\title{
Triggering of apoptosis by cathepsins
}

\author{
M Leist ${ }^{\star, 1}$ and $M$ Jäättelä ${ }^{2}$ \\ ${ }^{1}$ H. Lundbeck A/S, Ottiliavej 9, DK-2500 Valby, Denmark \\ 2 Apoptosis Laboratory, Danish Cancer Society, Strandboulevarden 49, \\ DK-2100 Copenhagen, Denmark \\ * Corresponding author: E-mail: male@lundbeck.com
}

Two recent papers ${ }^{1,2}$ have expanded the role of the proteolytic mediators of apoptotic cell death beyond caspases. The newly discovered proteases capable of triggering pro-apoptotic changes in mitochondria are in fact old-fashioned proteins confined to the lysosomes and known for their involvement in non-specific cellular waste disposal, i.e. the cathepsins.

'Knife and fork are evolutionary optimized tools to dine in a neat and efficient way'. Using this analogy of dining for cell demise and cutlery for caspases, two evident questions arise: are knife and fork sufficient tools in all cases - and, are they always necessary? The first question brings to mind an array of other sophisticated tools developed for eating specific foods like e.g. lobster or fondue. In the cell death world such additional gadgets could correspond to other proteases like the above mentioned cathepsins. ${ }^{3}$

Formerly, cathepsins were thought to lead to cellular autolysis and damage of neighboring cells during necrosis (Figure 1). Accordingly, lysosomes were regarded as 'suicide bags' that would release unspecific digestive enzymes after rupturing during uncontrolled cellular stress. Recent in vitro ${ }^{4-6}$ as well as in vivo ${ }^{7}$ data suggest, however, that cathepsins may also act as mediators of programmed cell death. The fact that the frequently used caspase inhibitor zVAD-fmk efficiently blocks cysteine cathepsins like cathepsin-B (cathB), ${ }^{8}$ further emphasizes the need for a re-evaluation of the existing literature on the relative roles of caspases and cathepsins in a number of apoptosis paradigms.

How is it possible that a specific cell death program can be triggered by the rather unspecific digestive power of lysosomal proteases? It appears as if a specific translocation process could be a key to the understanding of this phenomenon. For instance the selective translocation of cathB from lysosomes to cytosol and nucleus is well documented for bile salt-induced hepatic apoptosis. ${ }^{5}$ Similarly, there is clear evidence of early cathepsin-D translocation from secondary lysosomes to the cytosol under conditions of oxidative stress induced apoptosis. ${ }^{9,10}$ A second possibility is a quantitative relationship between the amount of lysosomal rupture and the mode of cell death. According to this model, low stress intensities trigger a limited release of lysosomal enzymes to the cytoplasm followed by apoptotic death, while high intensity stresses would lead to generalized lysosomal rupture and rapid cellular necrosis. ${ }^{11} \mathrm{~A}$ causal association between a limited lysosomal rupture and apoptosis has been supported by experiments showing cytoprotection by membrane stabilizing agents ${ }^{9}$ as well as triggering of cell death by selective lysosomal disrupters. ${ }^{12}$

The work by Guicciardi et al. from G. Gores' laboratory now provides some powerful evidence for the causal involvement of cathB in the caspase-8-dependent apoptotic signaling cascade triggered by TNF in vivo. ${ }^{1}$ The main conclusions rely on the comparisons of cathB-deficient mice with wild-type animals. For in vitro or in vivo experiments, hepatocytes were rendered sensitive to the apoptosis-inducing TNF signals by adenoviral transfer of an $I_{\kappa} \mathrm{B}$ super-repressor. When treated afterwards with TNF, liver damage in cathB-deficient mice was reduced, survival was increased and apoptotic markers of cultured hepatocytes from such mice were reduced compared to the similarly treated wild-type controls. Additional mechanistic studies revealed a release of cathB to the cytosol following TNF exposure. Here, the question arises whether cathB activity is sufficiently stable at the neutral intracellular $\mathrm{pH}$ to trigger downstream apoptotic processes in the cytosol. CathB is inactivated rapidly in neutral buffers in vitro, and, consistent with its normal localization within an acidic organelle, its dipeptidylpeptidase activity has an optimum at around $\mathrm{pH} 5$. However, biological fluids seem to have a stabilizing effect on cathB even at neutral $\mathrm{pH}$, and, in fact, cathB's endopeptidase activity has its optimum at $\mathrm{pH} 7.4$ (M. Leist and M. Jäättelä, unpublished observations; reviewed $\left.i^{13}\right)$. Accordingly, Guicciardi et al. demonstrated that cathB can trigger cytochrome $c$ release from mitochondria in an in vitro system, providing that a cytosolic extract was also added. This places cathB within the TNF-triggered apoptotic cascade as an enhancer acting upstream of mitochondria. Consistent with this, further experiments in a cell free system showed a release of cathB from lysosomes during incubation with caspase-8. The question on whether this mechanism is relevant within cells awaits further elucidation, since cathB does not seem to take a major role in another caspase-8-dependent pathway within the same hepatocytes, i.e. CD95-triggered apoptosis (M. Guicciardi, unpublished observations).

An elegant study by Stoka et al. coordinated by G. Salvesen complements the above in vivo data by addressing the potential biochemical mechanism of

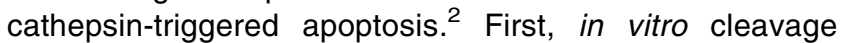
experiments showed that the major human apoptosisrelevant caspases are poor direct targets for lysomal extracts or a number of cysteine cathepsins. A possibility for a direct caspase activation was, however, indicated by data showing that a trypanosomal cysteine cathepsin (cruzipain, related to human cathL) can activate caspases-3/7. This corroborates previous observations of direct caspase-3 activation by a human cathL-like 


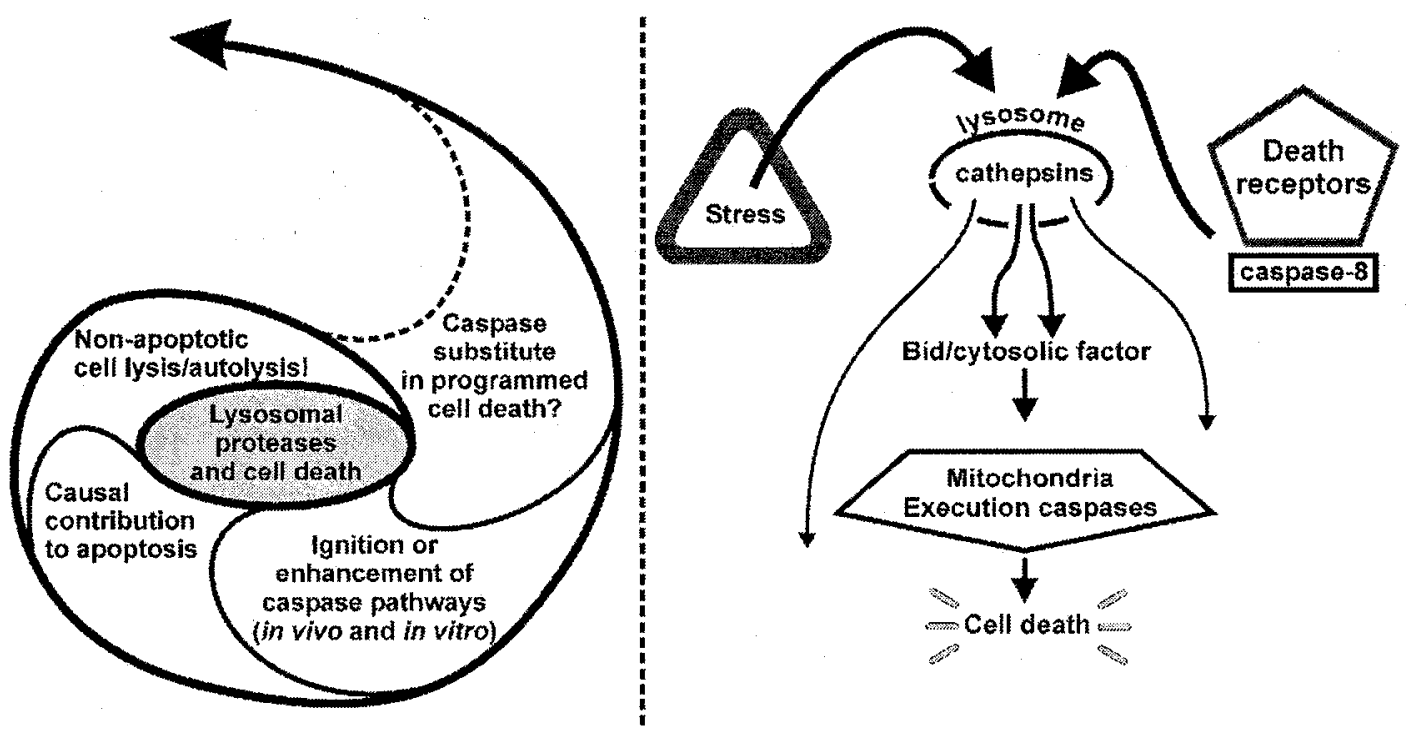

Figure 1 New roles of cathepsins in cell death. (Left) The evolving view of the role of cathepsins in cell death: severe insults may result in massive lysosomal rupture and autolysis of the cell as well as damage of surrounding tissue by released cathepsins. More recently, involvement of cathepsins in apoptosis has been discussed. Two topical studies ${ }^{1,2}$ demonstrate a possible mechanism for indirect triggering of caspases by cathepsins. Circumstantial evidence further suggests that cathepsins may take over some of the roles initially ascribed to caspases. (Right) Current evidence suggests that either triggering of TNF receptors or mild cellular stress initiate the release of cathepsins from lysosomes. After translocation, they may cleave cytosolic substrates like Bid, trigger the release of mitochondrial factors, and eventually evoke apoptotic cell death

protease. ${ }^{14}$ A further example of direct caspase processing by cathepsins is the activation of the inflammatory caspase11 by cathB. ${ }^{15}$ Since the quantitative issue on how much caspase activation is required for the apoptotic/inflammatory cascade to run to the end is still unresolved, more in vivo studies will be required for probing the relevance of direct caspase activation (or inactivation) by other proteases. Leaving this question aside, Stoka et al. present a striking new explanation for apoptosis activation by cathepsins. They demonstrate that the pro-apoptotic Bcl2 family member Bid is cleaved at the peptide loop joining the p15 and p7 subunits by lysosomal proteases in an in vitro system combining mitochondria and cytosol. ${ }^{2}$ Thus, very much in analogy to the cleavage by caspase-8, a Bid species is created that can potently trigger cytochrome $c$ release from mitochondria. The authors go further in their experiments by demonstrating that Bid cleavage is in fact the major event leading to cathepsin-mediated cytochrome $c$ release, since cytosol from Bid deficient mice was hardly functional in their assay.

The most appealing conclusion bringing together the in vitro $^{2}$ and the in vivo ${ }^{1}$ studies discussed above would be a cathB-mediated cleavage of Bid which would then trigger cytochrome $c$ release and activation of downstream caspases (Figure 1). This will, however, have to be put under experimental scrutiny, since the current in vitro data on human cathB do not support direct Bid proteolysis. ${ }^{2}$ Additional experiments will also be required to define the cell types that may be able to activate cathB-dependent apoptosis-enhancing loops, and to define their contribution with respect to other proteases involved in the death cascade.
If the data of Stoka prove true in vivo, and cathepsins can in fact cleave the essential caspase substrate Bid in an apoptosis-specific manner, other pro-apoptotic proteins may also be cathepsin substrates. In fact, in addition to the recently unveiled upstream effects of cathepsins, strong evidence has previously been provided that cathB can play a crucial role downstream of caspases, e.g. in bile saltinduced apoptosis. A similar proteolytic overlap has been suggested for caspases and calpains. ${ }^{16}$ Thus, one may envisage alternative proteolytic events in part or entirely substituting caspase action. In fact. P. Vandenabeele and others have shown that TNF or CD95 can signal programmed cell death independent of caspases. ${ }^{17}$ At least in tumor cells, part of these caspase-independent death signals are brought about by cathB (M. Leist and M. Jäättelä, unpublished observations). In pathological situations the cytochrome c/caspase pathway following opening of the mitochondrial permeability barrier by cathB may be precluded by ATP-deficiency, ${ }^{18}$ and nuclear apoptosis may be executed by $\mathrm{AIF}^{19}$ or direct effects of cathB on the nucleus. ${ }^{6}$ This closes the circle to the introduction, and brings us back to the answer for the second dining question on the absolute necessity of knife and fork: only a part of the world population uses knife and fork for eating, whereas many rely on other tools like sticks!

\section{References}

1. Guicciardi ME et al. (2000) J. Clin Invest. 106: 1127-1137

2. Stoka V et al. (2001) J. Biol. Chem. 276: 3149-3157

3. Johnson DE (2000) Leukemia 14: 1695-1703 
4. Deiss LP et al. (1996) EMBO J. 15: 3861-3870

5. Roberts LR et al. (1999) Cell Biochem. Biophys. 30: 71-88

6. Vancompernolle $\mathrm{K}$ et al. (1998) FEBS Lett. 438: 150-158

7. Yamashima T et al. (1998) Eur. J. Neurosci. 10: 1723-1733

8. Schotte $P$ et al. (1999) FEBS Lett. 422: 117-121

9. Roberg K and Öllinger K (1998) Am. J. Pathol. 152: 1151-1156

10. Roberg K et al. (1999) Free Radic. Biol. Med. 27: 1228-1237

11. Brunk UT et al. (1997) Free Radic. Biol. Med. 23: 616-626
12. Li W et al. (2000) FEBS Lett. 470: 35-39

13. Linebaugh BE et al. (1999) Eur. J. Biochem. 264: 100-109

14. Ishisaka $R$ et al. (1999) Cell Struct. Funct. 24: 465-470

15. Schotte $P$ et al. (1998) Biochem. Biophys. Res. Commun. 251: 379-387

16. Wang KK (2000) Trends Neurosci. 59: 20-26

17. Fiers W et al. (1999) Oncogene 18: 7719-7730

18. Leist M et al. (1999) Exp. Cell Res. 249: 396-403

19. Susin SA et al. (2000) J. Exp. Med. 192: 571-580 